\title{
NUMERICAL MODELING OF ABRUPT HETEROJUNCTIONS USING A THERMIONIC-FIELD EMISSION BOUNDARY CONDITION
}

\author{
Kyounghoon Yang, Jack R. East and George I. Haddad \\ Solid State Electronics Laboratory, Department of Electrical Engineering and Computer Science, \\ The University of Michigan, Ann Arbor, MI 48109-2122, U.S.A.
}

(Received 31 July 1992; in revised form I September 1992)

\begin{abstract}
We present a numerical model in which the thermionic and tunneling mechanisms across an abrupt heterojunction interface are taken into account on the basis of the one dimensional drift-diffusion formulation. We use an expression of thermionic-field emission current formulated based on the WKB approximation as a boundary condition at the abrupt heterointerface which eventually limits the current transport over the barrier while maintaining the current continuity. The $I-V$ characteristics of three types of $\mathrm{GaAs} / \mathrm{AlGaAs}$ heterojunctions are analyzed by varying device dimension, doping density, and temperature and compared with those obtained by the thermionic emission model to illustrate the significance of both tunneling and thermionic emission mechanisms. We demonstrate that the role of tunneling in the overall current transport is very important in these abrupt heterojunctions especially at high doping densities and low temperatures. In the case of an MBE-grown AlGaAs triangular heterojunction barrier, the temperature-dependent $I-V$ characteristics are measured and compared with the theoretical results. Good agreement is obtained when the tunneling process is taken into account by employing the thermionic-field emission boundary condition presented here.
\end{abstract}

\section{INTRODUCTION}

As modern epitaxial technologies such as MBE, MOCVD, and MOMBE make it possible to realize almost any doping and compositional profile of compound semiconductors with precise control of thickness, excellent device performance has been demonstrated in a wide variety of heterostructure devices. To aid in understanding the operational mechanisms of these devices and to optimize their design, various computational methods have been developed[1-4]. Among them, numerical simulations based on the drift-diffusion transport, known as the best approach for evaluating generation-recombination processes in the device structure, have been widely applied for the characterization of heterostructure devices[4-8]. Although the generalized drift-diffusion transport equations formulated for materials with a position-dependent band structure have been used, the validity of this formulation at abrupt heterojunctions is questionable.

Recently, Horio and Yanai[9] presented a more accurate numerical technique by taking into account the thermionic emission process at the abrupt heterojunction interface in conjunction with the drift-diffusion formulation for the bulk region. The thermionic emission current formulated at the heterointerface is used as a boundary condition which constrains the current transport across the interface. The effects of the emission mechanism on the $I-V$ characteristics have been found significant especially for $n N$ isotype heterojunctions. However, since the thermionic emis- sion mechanism describes only the carrier transport over the heterojunction barrier, this model can not be applied for a case where the tunneling process becomes important. Therefore, a more generalized numerical model is required.

In this paper, we present a numerical model in which thermionic emission as well as tunneling across the heterointerface is taken into account by developing a thermionic-field emission boundary condition. This boundary condition is formulated based on the WKB approximation and the detail derivation is described in the following section. In Section 3, we apply this model for the GaAs/AlGaAs $n N$ and $p N$ heterojunctions and illustrate the relative significance of both thermionic emission and tunneling as a function of device dimension, doping density, and temperature. Finally, the temperature dependent $I-V$ characteristics of an MBE-grown AlGaAs triangular heterojunction barrier are measured and compared with the theoretical results.

\section{NUMERICAL MODEL}

\subsection{Formulation of a thermionic-field emission boundary condition}

The band diagram of an abrupt heterojunction is shown in Fig. 1. Electron transport across the conduction band spike can be described by tunneling through the spike and thermionic emission over it. Using the one-dimensional WKB approximation[10], 


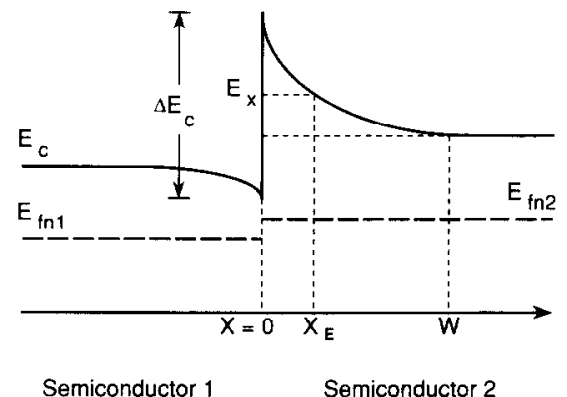

Fig. 1. Schematic band diagram of an abrupt heterojunction. $E_{f n \mid .2}$ represents the electron quasi-Fermi level in each semiconductor region.

the transmission probability of electrons for the barrier is expressed as follows:

$T\left(E_{x}\right)= \begin{cases}\exp \left(-\frac{4 \pi}{h} \int_{0}^{x_{\mathrm{E}}}\left[2 m_{n}^{*}\left\{E_{\mathrm{c}}(x)-E_{x}\right\}\right]^{1 / 2} \mathrm{~d} x\right), \\ \quad \text { if } E_{\min } \leqslant E_{x}<E_{\mathrm{c}}\left(0^{+}\right) \\ 1, \quad \text { if } E_{\mathrm{c}}\left(0^{+}\right) \leqslant E_{x},\end{cases}$

where $h$ is the Planck constant, $m_{n}^{*}$ is the electron effective mass, $E_{x}$ is the energy component in the $x$ direction, and $E_{\min }=\max \left[E_{\mathrm{c}}\left(0^{-}\right), E_{\mathrm{c}}(W)\right]$.

Assuming the Boltzmann energy distribution, the electron current density $J_{n}$ across the heterointerface arising from the net exchange of electrons is given by:

$$
\begin{aligned}
J_{n}=J_{n_{1}}-J_{n_{2}}= & -\frac{A^{*} T}{k} \int_{E_{\min }}^{x} f_{1}\left(E_{\mathrm{r}}\right) T\left(E_{x}\right) \mathrm{d} E_{x} \\
& +\frac{A^{*} T}{k} \int_{E_{\min }}^{x} f_{2}\left(E_{\mathrm{r}}\right) T\left(E_{\mathrm{r}}\right) \mathrm{d} E_{\mathrm{x}},
\end{aligned}
$$

where $k$ is the Boltzmann constant, $A^{*}$ is the effective Richardson constant for electrons, and $f_{1.2}\left(E_{x}\right)$ is the occupation probability in each region. The smaller value of effective mass between the two semiconductors is used for $A^{*}$ as was discussed in Ref. [11].

Using (1), we find:

$$
\begin{aligned}
J_{n_{1}}\left(0^{-}\right)= & -A^{*} T^{2} \exp \left(\frac{E_{j n_{1}}\left(0^{-}\right)-E_{\mathrm{c}}\left(0^{+}\right)}{k T}\right) \\
& \times\left[1+\frac{\exp \left(\frac{E_{\mathrm{c}}\left(0^{+}\right)}{k T}\right)}{k T} \int_{E_{\min }}^{E_{\mathrm{c}}\left(0^{+}\right)} \exp \left(-\frac{E_{x}}{k T}\right)\right. \\
& \times \exp \left(-\frac{4 \pi}{h} \int_{0}^{X_{\mathrm{E}}}\right. \\
& \left.\left.\times\left\{2 m_{n}^{*}\left[E_{\mathrm{c}}(x)-E_{x}\right]\right\}^{1 / 2} \mathrm{~d} x\right) \mathrm{~d} E_{x}\right] \\
= & -q v_{n_{1}}(1+\delta) n_{1}\left(0^{-}\right) \exp \left(-\frac{\Delta E_{\mathrm{c}}}{k T}\right)
\end{aligned}
$$

where:

$$
\begin{gathered}
n_{1}\left(0^{-}\right)=N_{\mathrm{c}_{1}} \exp \left(\frac{E_{f n_{1}}\left(0^{-}\right)-E_{\mathrm{c}}\left(0^{-}\right)}{k T}\right) \\
v_{n_{1}}=\frac{A^{*} T^{2}}{q N_{\mathrm{c}_{1}}} \\
\delta=\frac{\exp \left(\frac{E_{\mathrm{c}}\left(0^{+}\right)}{k T}\right)}{k T} \int_{E_{\min }}^{E_{\mathrm{c}\left(0^{+}\right)}} \exp \left(-\frac{E_{x}}{k T}\right) \\
\times \exp \left(-\frac{4 \pi}{h} \int_{0}^{X_{\mathrm{E}}}\left[2 m_{n}^{*}\left\{E_{\mathrm{c}}(x)-E_{x}\right\}\right]^{1 / 2} \mathrm{~d} x\right) \mathrm{d} E_{x} .
\end{gathered}
$$

A similar derivation yields the following equation for the opposing flux of electron current:

$$
J_{n_{2}}\left(0^{+}\right)=-q v_{n_{2}}(1+\delta) n_{2}\left(0^{+}\right),
$$

where:

$$
\begin{gathered}
n_{2}\left(0^{+}\right)=N_{\mathrm{c}_{2}} \exp \left(\frac{E_{f n_{2}}\left(0^{+}\right)-E_{\mathrm{c}}\left(0^{+}\right)}{k T}\right) \\
v_{n_{2}}=\frac{A^{*} T^{2}}{q N_{\mathrm{c}_{2}}} .
\end{gathered}
$$

Here, $v_{n, 2}$ is the mean electron thermal velocity, $N_{c_{1,2}}$ is the effective density of states in the conduction band in each region, and $n_{1}\left(0^{-}\right)$and $n_{2}\left(0^{+}\right)$are the electron densities at each side of the heterointerface.

Finally, the net electron current density crossing at the heterointerface is:

$$
\begin{aligned}
J_{n, i}=- & q v_{n_{1}}(1+\delta) n_{1}\left(0^{-}\right) \\
& \times \exp \left(-\frac{\Delta E_{c}}{k T}\right)+q v_{n_{2}}(1+\delta) n_{2}\left(0^{+}\right) .
\end{aligned}
$$

As can be seen in eqn (10), the thermionic-field emission process is taken into account through a parameter $\delta$ and, when the tunneling mechanism is neglected $(\delta=0)$, this equation becomes the thermionic emission boundary condition similar to that used in Ref. [9].

For holes, the thermionic emission boundary condition is used at the interface since the $n N$ and $p N$ heterojunctions simulated here have the tunneling barriers only in the conduction band and in the case of a triangular heterojunction barrier created between layers of $n$-type GaAs the contribution of tunneling by holes is negligible.

The thermionic emission boundary condition for holes is:

$$
J_{p, i}=q v_{p_{1}} p_{1}\left(0^{-}\right) \exp \left(-\frac{\Delta E_{v}}{k T}\right)-q v_{p_{2}} p_{2}\left(0^{+}\right)
$$

where:

$$
\begin{aligned}
& v_{p_{1}}=\frac{A_{\mathrm{h}}^{*} T^{2}}{q N_{r_{1}}} \\
& v_{p_{2}}=\frac{A_{\mathrm{h}}^{*} T^{2}}{q N_{v_{2}}} .
\end{aligned}
$$


Here, $A_{h}^{*}$ is the effective Richardson constant for holes, $v_{p_{1,2}}$ is the mean hole thermal velocity, $N_{v_{1,2}}$ is the effective density of states in the valence band in each region, and $p_{1}\left(0^{-}\right)$and $p_{2}\left(0^{+}\right)$are the hole densities at each side of the heterointerface.

In the present work, we use the current expressions of eqns (10) and (11) as the boundary conditions for the electron and hole currents at the abrupt heterointerface and apply the conventional driftdiffusion model in the remainder of the structure where the material composition is constant, or slowly varying. This approach can resolve several difficulties encountered when we attempt to couple two different schemes of transport formulation, tunneling-emission and drift-diffusion, in a device structure. By incorporating the tunneling mechanism only at one mesh point of the interface, the difficulty of current formulation in the energy barrier region where the combined transport phenomena of tunneling and drift-diffusion mechanisms exist is avoided and the recombination-generation process can be evaluated over the whole device structures. Although the tunneling parameter $\delta$ is used only at a mesh point, the barrier transparency represented by $\delta$ is evaluated based on the overall conduction band profile at each bias.

The problem solution begins by using the thermionic emission boundary conditions $(\delta=0)$ for both electrons and holes in which all parameters such as thermal velocities, and the conduction and valence band discontinuities are already known. The relation between the quasi-Fermi levels of both sides are self-consistently determined by the boundary conditions. After the first calculation is completed with the thermionic emission condition, the value of $\delta$ is evaluated using eqn (6) based on the obtained conduction band profile and then the thermionic-field emission boundary condition of eqn (10) is employed in the following calculation steps. In each iteration, the energy band profiles and the electron and hole quasi-Fermi levels are updated by solving the Poisson equation, the electron and hole current continuity equations self-consistently. This process continues until the change in $\delta$ is less than $0.1 \%$. Five iterations are typically enough to obtain the final solution after the first calculation with the thermionic emission boundary conditions is achieved.

\subsection{Basic equations of the drift-diffusion model}

In the present work, the basic equations based on the drift-diffusion model are employed for the region where the material composition changes slowly. For one-dimensional steady-state, the equations are expressed as follows:

$$
\begin{aligned}
\frac{\mathrm{d}}{\mathrm{d} x}\left(\epsilon \frac{\mathrm{d} \psi}{\mathrm{d} x}\right) & =-q\left(p-n+N_{\mathrm{D}}^{+}-N_{\mathrm{A}}^{-}\right) \\
\frac{\mathrm{d} J_{n}}{\mathrm{~d} x} & =q(R-G)
\end{aligned}
$$

$$
\frac{\mathrm{d} J_{p}}{\mathrm{~d} x}=-q(R-G)
$$

and

$$
\begin{gathered}
J_{n}=-q \mu_{n} n \frac{\mathrm{d}}{\mathrm{d} x}\left(\psi+\psi_{n}\right)+k T \mu_{n} \frac{\mathrm{d} n}{\mathrm{~d} x} \\
J_{p}=-q \mu_{p} p \frac{\mathrm{d}}{\mathrm{d} x}\left(\psi-\psi_{p}\right)-k T \mu_{p} \frac{\mathrm{d} p}{\mathrm{~d} x} \\
\psi_{\mathrm{n}}=\frac{\chi(x)-\chi_{\mathrm{r}}}{q}+\frac{k T}{q} \ln \left(\frac{N_{\mathrm{c}}(x)}{N_{\mathrm{cr}}}\right) \\
\psi_{p}=\frac{-\chi(x)-\chi_{\mathrm{r}}}{q}-\frac{E_{\mathrm{g}}(x)-E_{\mathrm{gr}}}{q}+\frac{k T}{q} \ln \left(\frac{N_{\mathrm{v}}(x)}{N_{\mathrm{vr}}}\right)
\end{gathered}
$$

where $\psi$ denotes the electrostatic potential, $\psi_{n}$ and $\psi_{p}$ are composition dependent band parameters, and $\chi$ is the electron affinity. Other symbols have their standard meanings. The subscript $r$ represents the parameter values at a reference point in the device structure. The term $R$ denotes the recombination rate and is assumed to be specified by the ShockleyRead-Hall model:

$$
R=\frac{p n-n_{\mathrm{i}}^{2}}{\tau_{n 0}\left(p+n_{\mathrm{i}}\right)+\tau_{p 0}\left(n+n_{\mathrm{i}}\right)},
$$

where $n_{\mathrm{i}}$ is the intrinsic carrier density and $\tau_{\mathrm{n} 0}$ and $\tau_{p 0}$ are life times for electrons and holes, respectively.

The incomplete ionization of impurities is taken into account by the following formulas:

$$
\begin{aligned}
& N_{\mathrm{D}}^{+}=\frac{N_{\mathrm{D}}}{1+\frac{n}{N_{\mathrm{c}}} g_{\mathrm{D}} \exp \left(\frac{\Delta E_{\mathrm{D}}}{k T}\right)}, \\
& N_{\mathrm{A}}^{-}=\frac{N_{\mathrm{A}}}{1+\frac{p}{N_{\mathrm{v}}} g_{\mathrm{A}} \exp \left(\frac{\Delta E_{\mathrm{A}}}{k T}\right)},
\end{aligned}
$$

where $N_{\mathrm{D}}, N_{\mathrm{A}}$ are donor and acceptor impurity concentrations, $g_{\mathrm{D}}, g_{\mathrm{A}}$ are ground state degeneracy factors of the donor and acceptor level, and $\Delta E_{\mathrm{D}}$, $\Delta E_{\mathrm{A}}$ are the donor and acceptor activation energies. The degenerate factor for donors $g_{\mathrm{D}}$ is two, whereas that for holes $g_{\mathrm{A}}$ is four. The acceptor activation energy $\Delta E_{\mathrm{A}}$ is taken as $0.026 \mathrm{eV}$ (as for $\mathrm{Be}$ ) and the donor activation energy $\Delta E_{\mathrm{D}} 0.005 \mathrm{eV}$ (as for $\mathrm{Si}$ ) in GaAs. In AlGaAs, the donor activation energy depends on the aluminum mole fraction[12]. For $\mathrm{Al}_{0.25} \mathrm{Ga}_{0.75} \mathrm{As}$ used as a larger band gap semiconductor in the $n N$ and $p N$ heterojunctions, $0.018 \mathrm{eV}$ is taken as $\Delta E_{\mathrm{D}}$.

In the present work, the temperature-dependent material parameters of the energy bandgap, and electron and hole mobilities are used.

For GaAs, the energy bandgap at the $\Gamma$ point[13], and the low-field mobilities for the electrons and holes[14] are expressed as follows:

$$
E_{\mathrm{g} \Gamma}=1.519-5.405 \times 10^{-4} \frac{T^{2}}{(T+204)} \mathrm{eV},
$$




$$
\begin{aligned}
& \mu_{n}=\frac{7200}{\left[1+5.51 \times 10^{-17}\left(N_{\mathrm{D}}+N_{\mathrm{A}}\right)\right]^{0.233}} \\
& \times\left(\frac{300}{T}\right)^{2.3} \mathrm{~cm}^{2} / \mathrm{Vs} \\
& \mu_{p}=\frac{380}{\left[1+3.17 \times 10^{-17}\left(N_{\mathrm{D}}+N_{\mathrm{A}}\right)\right]^{0.266}} \\
& \times\left(\frac{300}{T}\right)^{2.7} \mathrm{~cm}^{2} / \mathrm{Vs} \text {. }
\end{aligned}
$$

The electric field dependence of mobilities is not considered for simplification. For $\mathrm{Al}_{x} \mathrm{Ga}_{1-x} \mathrm{As}$ $(0<x<0.45)$, the temperature-dependence of $E_{\mathrm{g} I}$ is assumed to be the same as that of GaAs, and the electron and hole mobilities are reduced by 12.7 and $6.7 \%$ per aluminum composition of 0.1 from the values in GaAs, respectively. The other material parameters utilized in the present work are listed in Table 1 as a function of the aluminum mole fraction $x$.

\section{NUMERICAL ANALYSIS RESULTS}

The numerical model described in the previous section is applied to the three examples of GaAs/ AlGaAs abrupt heterostructure devices shown in Fig. 2; an $n N$ isotype heterojunction, a $p N$ anisotype heterojunction, and a triangular heterojunction barrier diode. The current-voltage characteristics are analyzed as a function of doping density, device dimension and temperature using both the thermionic emission and thermionic-field emission models to illustrate the relative significance of thermionic emission and tunneling transport mechanisms.

\subsection{An n-GaAs $/ N-A l_{0.25} G a_{0.75}$ As heterojunction}

In this section, carrier transport across the isotype $n N$ heterojunctions is investigated. The studies on the $n N$ heterojunctions have demonstrated that these devices display rectifying current vs voltage characteristics[15,16].

First, the effects of the doping densities on the $I-V$ characteristics are studied. The theoretical $I-V$ characteristics of an $n N$ heterojunction for three different doping densities of $N_{\mathrm{D}_{2}}$ are shown in Fig. 3. Here, the doping density of semiconductor 1 is kept constant. The solid lines and the dashed lines represent the thermionic-field emission and the thermionic emission model, respectively. For $N_{\mathrm{D}_{2}}=10^{15}$ $\mathrm{cm}^{-3}$, little difference is observed between the two

Table 1. Material parameters used in the simulation

\begin{tabular}{ll}
\hline \multicolumn{1}{c}{ Parameter } & \multicolumn{1}{c}{ Formula $(0 \leqslant x<0.45)$} \\
\hline Band gap (eV) & $E_{\mathrm{g} r}=E_{\mathrm{g} r, \mathrm{GaAs}_{\mathrm{s}}+1.247 x}$ \\
Electron affinity (eV) & $\chi=4.07-0.6\left[E_{\mathrm{g} r}(x)-E_{\mathrm{g} r, \mathrm{GaAs}}\right]$ \\
Electron effective mass & $m_{n}^{*}=(0.067+0.083 x) m_{0}$ \\
Hole effective mass & $m_{p}^{*}=(0.48+0.31 x) m_{0}$ \\
Dielectric constant & $\epsilon=(13.1-3 x) \epsilon_{0}$ \\
Electron life time (s) & $\tau_{n 0}=10^{-9}$ \\
Hole life time (s) & $t_{\mathrm{p} 0}=10^{-9}$ \\
\hline
\end{tabular}

(a)

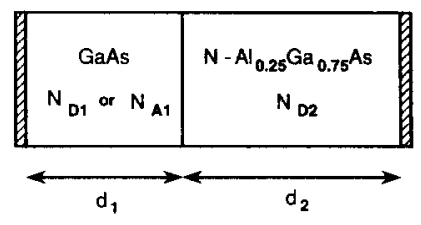

(b)

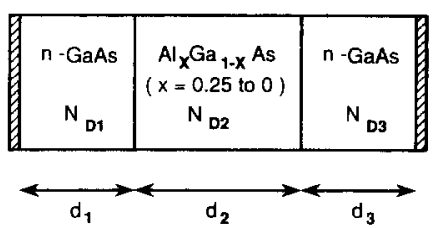

Fig. 2. The device structures used for (a) the $n N$ and $p N$ diodes and (b) the triangular heterojunction barrier diode

models. However, a significant contribution by tunneling is found at higher doping densities. The enhancement of current is large in the reverse bias region. When the device is forward biased, the conduction band edge of semiconductor 2 is shifted upward. With the decrease of energy barrier height, more electrons from side 2 go over the barrier rather than tunnel through it. However, under the reverse bias condition, the electrons injected from side 1 see an abrupt energy barrier whose height is determined by the conduction band discontinuity. In Fig. 4, the conduction band profiles of the $n N$ heterojunctions are plotted at each doping density of $N_{\mathrm{D}_{2}}$ at a reverse bias of $-0.1 \mathrm{~V}$. The electron quasi-Fermi level is shown for the case of $N_{\mathrm{D}_{2}}=5 \times 10^{16} \mathrm{~cm}^{-3}$. As is shown in the figure, at high doping densities, the notch of the conduction band gets close to the Fermi-level and the energy barrier gets thinner as well. The increase of reverse current with $N_{\mathrm{D}_{2}}$ predicted by the thermionic emission model can be described by the fact that the lowering of the effective

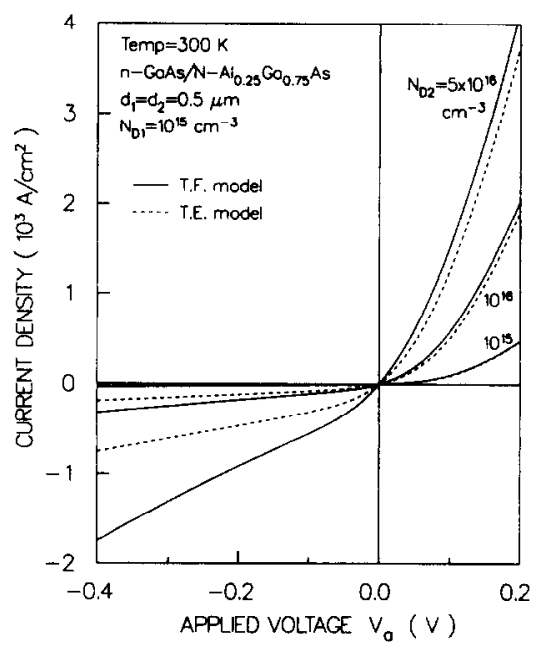

Fig. 3. Calculated $I-V$ characteristics of an $n-\mathrm{GaAs} / N$ $\Lambda \mathrm{I}_{0.25} \mathrm{Ga}_{0.75} \Lambda \mathrm{s}$ diode with $d_{1}=d_{2}=0.5 \mu \mathrm{m}$ and $N_{\mathrm{D}_{1}}=10^{15}$ $\mathrm{cm}^{-3}$ at different doping densities of $N_{\mathrm{D}_{2}}$. The solid and dashed lines represent the thermionic-field emission and thermionic emission model, respectively. 


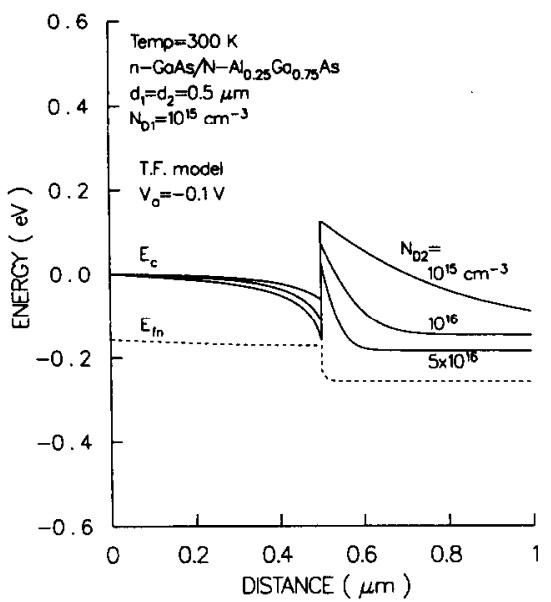

Fig. 4. Calculated conduction band profiles of the $n$ $\mathrm{GaAs} / N-\mathrm{Al}_{0.25} \mathrm{Ga}_{0.75}$ As diode at different doping densities of $N_{\mathrm{D}_{2}}$ under a bias of $-0.1 \mathrm{~V}, d_{1}=d_{2}=0.5 \mu \mathrm{m}$ and $N_{\mathrm{D}_{1}}=10^{15} \mathrm{~cm}^{-3}$.

barrier height helps more electrons go over the barrier. However, as the barrier thickness is reduced, the electrons with lower energy than the barrier can also contribute to the current conduction by tunneling through the barrier. For the case of $N_{\mathrm{D}_{2}}=5 \times 10^{16}$ $\mathrm{cm}^{-3}$, rectifying $I-V$ characteristics are not obvious any more due to significant enhancement of reverse current by tunneling (see Fig. 3). The discontinuity in the quasi-Fermi level shown at the interface indicates that the current transport over the barrier is limited by the thermionic-field emission mechanism rather than by drift-diffusion across the space charge region as discussed in Ref. [9]. Under appreciable reverse bias, the notch of the conduction band edge on the GaAs side may dip significantly below the Fermi level. In this situation, the actual barrier lowering will

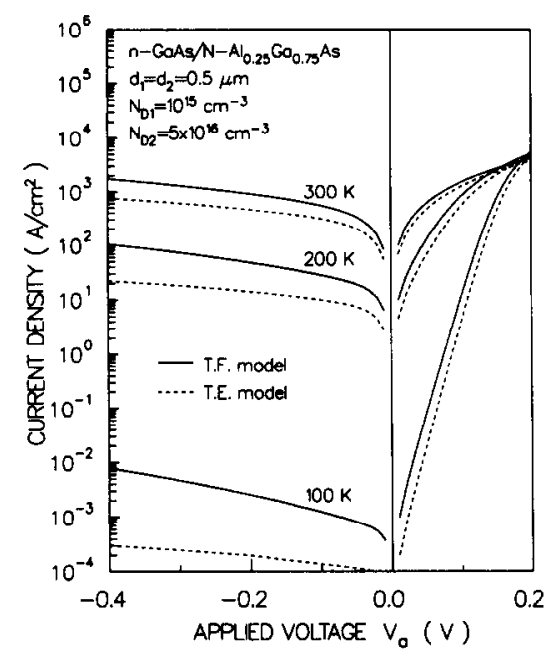

Fig. 5. Calculated $I-V$ characteristics of an $n-\mathrm{GaAs} / N$ $\mathrm{Al}_{0.25} \mathrm{Ga}_{0.75}$ As diode with $d_{1}=d_{2}=0.5 \mu \mathrm{m}, N_{\mathrm{D}_{1}}=10^{15} \mathrm{~cm}^{-3}$, and $N_{\mathrm{D}_{2}}=5 \times 10^{16} \mathrm{~cm}^{-3}$ at different temperatures using the thermionic-field emission model (solid line) and the thermionic emission model (dashed line). be greater than that estimated by the present model based on the Boltzmann distribution.

In the $n N$ heterojunetions, the transport of injected electrons in the quasi-neutral region does not limit the overall $I-V$ characteristics since the electrons are majority carriers in both semiconductor regions and the redistribution of injected electrons in the quasi-neutral region is a fast process. Therefore, the dimensions of the bulk regions have no significant impact on the $I-V$ characteristics except the corresponding ohmic voltage drops. However, the situation is different in the $p N$ heterojunctions. Here, the minority carriers after injection into the quasi-neutral regions either recombine with majority carriers, or diffuse away depending on the device dimension and recombination mechanisms. This will be discussed in greater detail later in the example of a $p N$ heterojunction.

Next, the $I-V$ characteristics of the $n N$ heterojunctions are analyzed by varying the temperature. Figure 5 shows the theoretical $I-V$ curves at $T=100,200$ and $300 \mathrm{~K}$. At low temperatures, the $I-V$ characteristics look more exponential because the ohmic voltage drops over the quasi-neutral regions are greatly reduced because of the increase of the electron and hole mobilities. It is also observed that the current conduction by tunneling becomes considerable as temperature decreases. In Fig. 6, the ratio between the current components of tunneling and thermionic emission is plotted as a function of temperature for two different doping densities. The tunneling current component is obtained by subtracting the thermionic emission current estimated by the thermionic emission model from the total current evaluated by the thermionic-field emission model. As was verified in the Schottky barrier diodes[17], the role of tunneling in the current conduction becomes

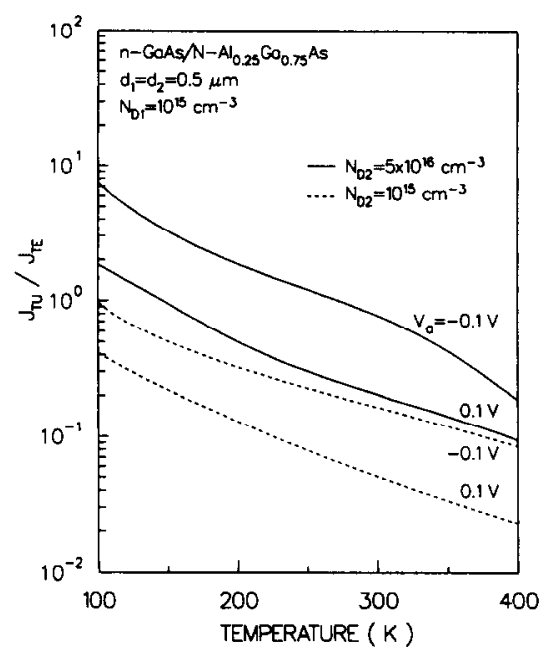

Fig. 6. The ratio of the tunnel current to thermionic current of the $n-\mathrm{GaAs} / N-\mathrm{Al}_{0.25} \mathrm{Ga}_{0.75}$ As diode with $d_{1}=d_{2}=0.5 \mu \mathrm{m}$ and $N_{\mathrm{D}_{1}}=10^{15} \mathrm{~cm}^{-3}$ for the case of $N_{\mathrm{D}_{2}}=10^{15} \mathrm{~cm}^{-3}$ (dashed line) and $5 \times 10^{16} \mathrm{~cm}^{-3}$ (solid line) at a bias of $V_{\mathrm{a}}=0.1 \mathrm{~V}$ and $-0.1 \mathrm{~V}$. 
more important as temperature is decreased and doping density is increased. For the case of $N_{\mathrm{D}_{2}}=5 \times 10^{16} \mathrm{~cm}^{-3}$, the portion of tunneling current over total current increases from 17 to $90 \%$ according to the decrease of temperature from 400 to $100 \mathrm{~K}$ at a bias of $-0.1 \mathrm{~V}$. These facts confirm that the tunneling mechanism must be taken into account when the electrical behavior of abrupt heterostructure devices is studied.

\subsection{A p-GaAs/N-A $l_{0.25} G a_{0.75}$ As heterojunction}

Compared to the $p n$ homojunctions of which transport theory was established by the drift-diffusion model, the study on the current conduction mechanisms of $p N$ heterojunctions has been semiquantitative[18-20]. This is mainly because we do not have a generalized theory which considers both tunnelingemission across the heterointerface and drift-diffusion in the quasi-neutral and space charge region. A $p N$ heterojunction diode shown in Fig. 2(a) is ana-

(a)

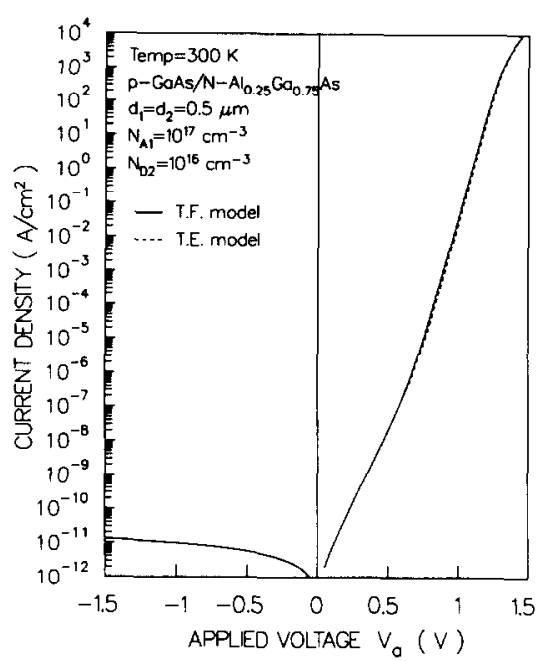

(b)

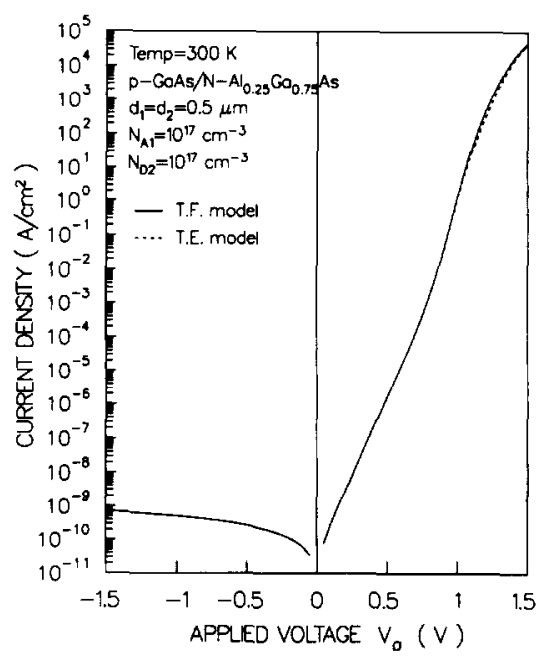

Fig. 7. Calculated $I-V$ characteristics of a $p-\mathrm{GaAs} / N$ $\mathrm{Al}_{0.25} \mathrm{Ga}_{0.75}$ As diode with $d_{1}=d_{2}=0.5 \mu \mathrm{m}$ for the case of (a) $N_{\mathrm{A}_{1}}=10^{17} \mathrm{~cm}^{-3}$ and $N_{\mathrm{D}_{2}}=10^{16} \mathrm{~cm}^{-3}$ and (b) $N_{\mathrm{A}_{1}}=10^{17}$ $\mathrm{cm}^{-3}$ and $N_{\mathrm{D}_{2}}-10^{17} \mathrm{~cm}^{-3}$. (a)

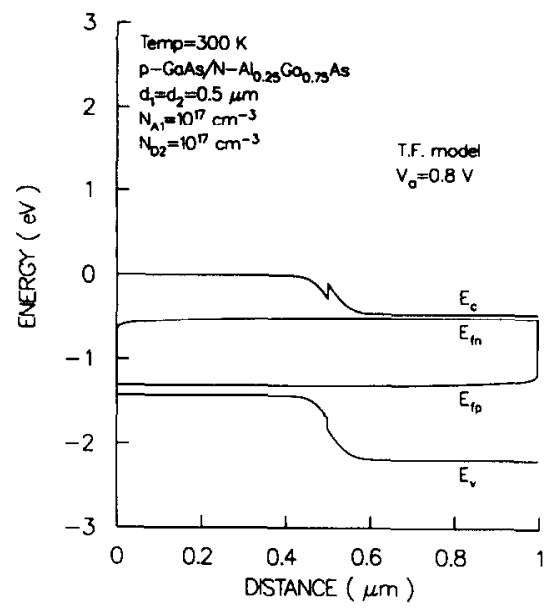

(b)

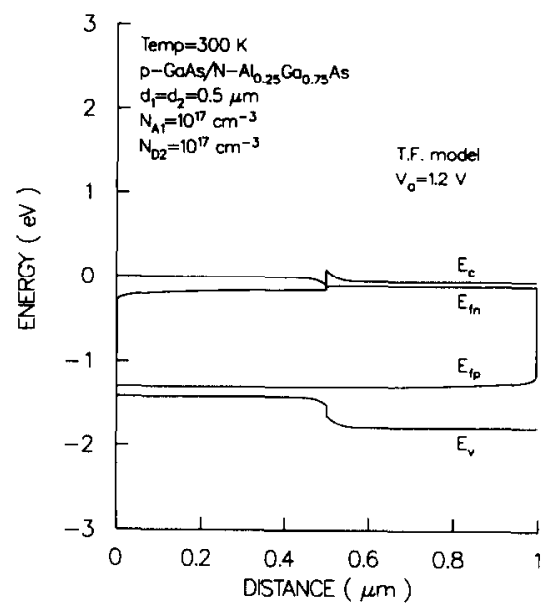

Fig. 8. Calculated energy band diagrams of the $p-\mathrm{GaAs} /$ $N-\mathrm{Al}_{0.25} \mathrm{Ga}_{0.75}$ As diode with $d_{1}=d_{2}=0.5 \mu \mathrm{m}$ and $N_{\mathrm{A}_{1}}=N_{\mathrm{D}_{2}}=10^{17} \mathrm{~cm}^{-3}$ at a bias of (a) $0.8 \mathrm{~V}$ and (b) $1.2 \mathrm{~V}$ using the thermionic-field emission model.

lyzed here by employing the present numerical model. In Fig. 7 , the $I-V$ characteristics of a $p N$ heterojunction diode with $d_{1}=d_{2}=0.5 \mu \mathrm{m}$ are shown at two different doping densities. In the low forward-bias region of the curves, the current conduction mechanism is characterized by the space charge recombination $\left[J \propto \exp \left(q V_{\mathrm{a}} / 2 k T\right)\right]$. In contrast to the $n N$ heterojunctions, the contribution of tunneling is observed only in the high forward bias region. This can be described by the fact that, in the $p N$ heterojunctions, a large number of electrons are supplied for current conduction only at high forward biases Under reverse bias, the net flow across the junction is composed of a few minority carriers from each region. With low forward bias, the electron injection across the interface is suppressed by a large electric field in the space charge region. Therefore, unless the device is highly forward biased, the current conduction over the heterojunction is limited by the carrier supply capability rather than by the injection mechanism of the interface. At a higher doping density of $N_{\mathrm{D}_{2}}=10^{17} \mathrm{~cm}^{-3}$, the contribution of tunneling is slightly enhanced and observed in the higher bias 
range because of the corresponding increase of the built-in potential. The energy band diagrams of a $p N$ heterojunction diode biased at $V_{\mathrm{a}}=0.8 \mathrm{~V}$ and $1.2 \mathrm{~V}$ are shown in Fig. 8. At $V_{\mathrm{a}}=0.8 \mathrm{~V}$, the electron energy barrier represented by the band bending across the space charge region is still high. So the effects of the conduction band spike on the electron injection across the interface are not significant. The continuous electron quasi-Fermi level at the interface indicates that the overall current is limited by driftdiffusion in the space charge region. However, under high forward bias, the spike projects above the conduction band in the $p$-type region, as shown in Fig. $8(\mathrm{~b})$, and starts to suppress the electron injection over the interface. This is confirmed by the discontinuity of the electron quasi-Fermi level found at the interface. In the case of the hole quasi-Fermi level, the discontinuity is not observed even under high forward bias. This indicates that, in the $p N$ heterojunctions, drift-diffusion in the space charge region limits

(a)

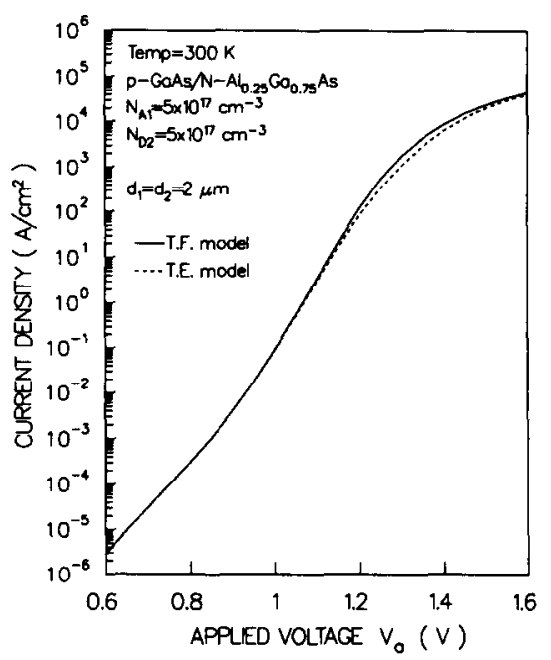

(b)

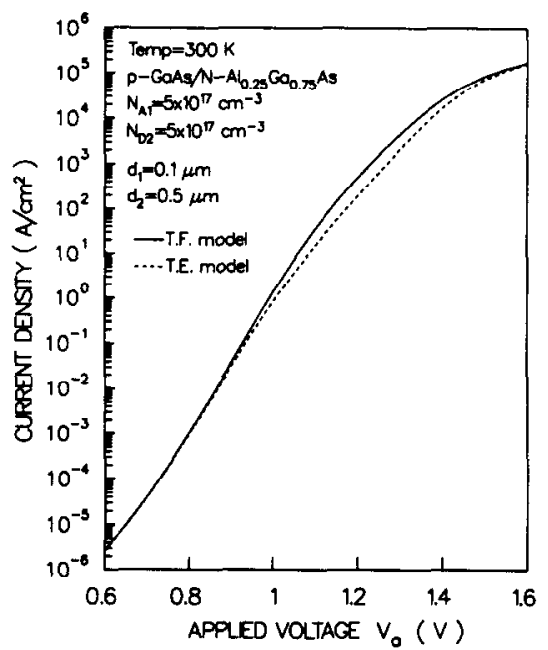

Fig. 9. Calculated forward $I-V$ characteristics of a $p$ $\mathrm{GaAs} / N-\mathrm{Al}_{0.25} \mathrm{Ga}_{0.75}$ As diode with $N_{\mathrm{A}_{1}}=N_{\mathrm{D}_{2}}=5 \times 10^{17}$ $\mathrm{cm}^{-3}$ for (a) $d_{1}=d_{2}=2 \mu \mathrm{m}$ and (b) $d_{1}=0.1 \mu \mathrm{m}$ and $d_{2}=0.5 \mu \mathrm{m}$. the hole transport across the junction. The hole injection into the $n$-type AlGaAs region is blocked by the abrupt valence band discontinuity for any bias condition.

Besides tunneling-emission across the interface and drift-diffusion in the space charge region, an additional conduction process of minority carrier diffusion in the quasi-neutral regions should be considered in the $p N$ heterojunctions. To illustrate the effects of minority carrier diffusion in the bulk region, the forward $I-V$ characteristics of the $p N$ diodes with different device lengths are compared in Fig. 9. For a thicker device of $d_{1}=d_{2}=2 \mu \mathrm{m}$, the contribution of tunneling is found less significant. When the length of the $p$-type region is much shorter than the electron diffusion length, the electron current by diffusion in the $p$-type region under low level injection is expressed as:

$$
J_{n}=q \frac{D_{p}}{W_{p}} \Delta n_{p}
$$

where $D_{p}$ is the diffusion constant of electrons in the $p$-type region, $W_{p}$ is the length of the quasi-neutral $p$-type region and $\Delta n_{p}$ is the excess electron density at the edge of the space charge region in the $p$-type semiconductor. At $300 \mathrm{~K}$, the electron diffusion length in the $p$-type region doped to $5 \times 10^{17} \mathrm{~cm}^{-3}$ is approximately $2.9 \mu \mathrm{m}$. Therefore, when the length of the $p$-type quasi-neutral region is shorter than this value, the overall electron transport is affected by the device dimensions. Assuming that ohmic drops in the quasi-neutral regions are small in the moderate forward bias region of Fig. 9, the difference in the contribution of tunneling found between two structures is attributed to the different device lengths since the diffusion constants are the same in both diode structures. As is demonstrated in this example, the current conduction of a $p N$ heterojunction is composed of three major transport processes, driftdiffusion in the space charge region (if the width of the space charge region becomes an order of a mean carrier free path, this process will not limit the carrier injection), carrier injection by tunneling and thermionic emission and minority carrier diffusion across the quasi-neutral regions.

Figure 10 shows the temperature-dependent $I-V$ characteristics of a $p N$ hetcrojunction. At low temperatures, considerable enhancement of current due to tunneling is observed over a wide range of applied bias. As temperature is decreased, the increase of electron and hole mobilities significantly reduces the contribution of ohmic voltage drop and enhances the minority carrier diffusion in the quasi-neutral regions. In Fig. 11, the ratio of the tunneling current component to thermionic emission component is plotted as a function of temperature for two different doping densities. As is expected, the carrier transport by the tunneling process becomes significant as the temperature decreases and doping density increases. In the case of $N_{\mathrm{A}_{1}}=N_{\mathrm{D}_{2}}=5 \times 10^{17} \mathrm{~cm}^{-3}$, the 


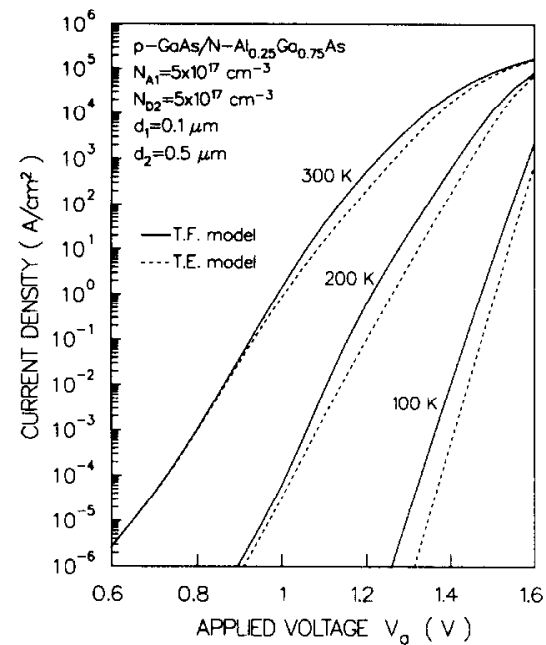

Fig. 10. Calculated forward $I-V$ characteristics of the $p$-GaAs $/ N-\mathrm{Al}_{0.25} \mathrm{Ga}_{0.75}$ As diode with $d_{1}=0.1 \mu \mathrm{m}, d_{2}=$ $0.5 \mu \mathrm{m}$, and $N_{\mathrm{A}_{1}}=N_{\mathrm{D}}=5 \times 10^{17} \mathrm{~cm}^{-3}$ at different temperatures. The solid and dashed lines represent the thermionicfield emission and thermionic emission model, respectively.

contribution by tunneling is 25 times as large as that of thermionic emission at a temperature of $100 \mathrm{~K}$ and an applied bias of $1.35 \mathrm{~V}$. These results clearly demonstrate that the effects of the tunneling process should be considered for the characterization of abrupt $p N$ heterojunction devices.

\section{3. $A n A l_{x} \mathrm{Ga}_{1-x} \mathrm{As}(x=0.25$ to 0$)$ triangular heterojunction barrier}

The current transport of a triangular heterojunction barricr shown in Fig. 2(b) is investigated in this section. This type of heterojunction barrier is known

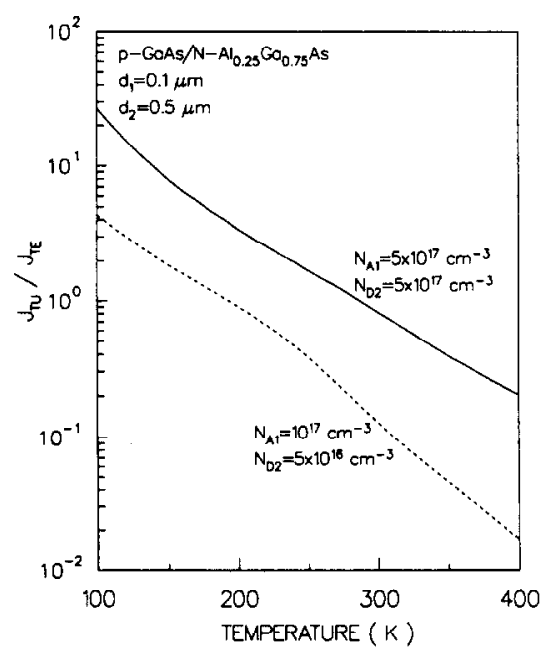

Fig. 11. The ratio of the tunnel current to thermionic current of the $p-\mathrm{GaAs} / N-\mathrm{Al}_{0.25} \mathrm{Ga}_{0.75}$ As diode with $d_{1}=0.1 \mu \mathrm{m}, \quad d_{2}=0.5 \mu \mathrm{m}$ for the case of $N_{\mathrm{A}_{1}}=$ $N_{\mathrm{D}_{2}}=5 \times 10^{17} \mathrm{~cm}^{-3}$ under a bias of $1.35 \mathrm{~V}$ (solid line) and $N_{\mathrm{A}_{1}}=10^{17} \mathrm{~cm}^{-3}$ and $N_{\mathrm{D}_{2}}=5 \times 10^{16} \mathrm{~cm}^{-3}$ under a bias of $1.4 \mathrm{~V}$ (dashed line).

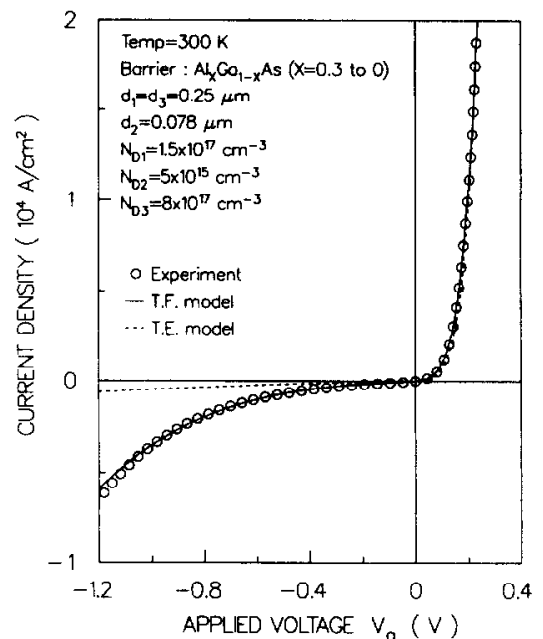

Fig. 12. The $I-V$ characteristics of an AlGaAs triangular heterojunction barrier diode at $300 \mathrm{~K}$. The circles represent the experimental results. The solid and dashed line show the thermionic-field emission and thermionic emission model, respectively.

to display rectifying $I-V$ characteristics similar to a Schottky barrier[21,22].

The temperature-dependent $I-V$ characteristics are measured using an MBE grown $\mathrm{Al}_{r} \mathrm{Ga}_{1-x}$ As graded heterojunction barrier and compared with theoretical results. The sample structure was grown on a (100) $\mathrm{GaAs}$ substrate by $\mathrm{MBE}$ using $\mathrm{Si}$ as the $n$-type dopant. A 20 period $30 \AA / 20 \AA \mathrm{GaAs} / \mathrm{Al}_{0.25} \mathrm{Ga}_{0.75}$ As superlattice doped $n^{+}=2 \times 10^{18} \mathrm{~cm}^{-3}$ was first grown as a buffer layer on the substrate $\left(n^{+}=2 \times 10^{18} \mathrm{~cm}^{-3}\right)$, followed by a $0.25 \mu \mathrm{m} \mathrm{GaAs}$ layer doped $n=2 \times 10^{17} \mathrm{~cm}^{-3}$, a $0.1 \mu \mathrm{m} \mathrm{Al}_{x} \mathrm{Ga}_{1-x}$ As barrier layer $\left(n=1 \times 10^{15} \mathrm{~cm}^{-3}\right)$ linearly graded from $x=0$ to 0.25 and followed by an abrupt composition discontinuity, a $0.25 \mu \mathrm{m}$ GaAs layer doped $n-2 \times 10^{17} \mathrm{~cm}^{-3}$, and finally a $0.5 \mu \mathrm{m}$ thick GaAs layer doped $n^{+}=2 \times 10^{18} \mathrm{~cm}^{-3}$ to aid in ohmic contact formation. Using the standad lift-off process, circular $20 \mu \mathrm{m}$ diameter mesa isolated diodes were fabricated and the temperature-dependent $I-V$ characteristics measured using an HP 4145B Semiconductor Parameter Analyzer and a Joule-Thomson expansion cooled cryostat. $\mathrm{Ni} / \mathrm{Au} / \mathrm{Ge} / \mathrm{Ti} / \mathrm{Au}$ ohmic contacts were made to the top and bottom of the sample.

The $I-V$ characteristics measured at $300 \mathrm{~K}$ (circles) are shown in Fig. 12 with the calculated results using both the thermionic emission (dashed line) and thermionic-field emission model (solid line). Only the active region of the device composed of layers of $\mathrm{GaAs} / \mathrm{Al}_{x} \mathrm{Ga}_{1-x} \mathrm{As} / \mathrm{GaAs}$ was taken in the simulation and the parameters used for fitting are specified in the caption. The donor activation energy of $0.035 \mathrm{eV}$ was taken for AlGaAs at the interface and assumed to vary linearly with the aluminum grading. As is shown in the figure, the measured $I-V$ characteristics of the triangular heterojunction barrier are 
(a)

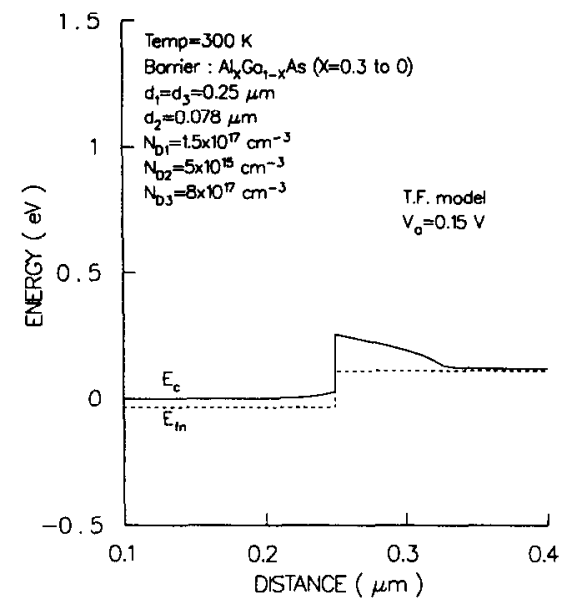

(b)

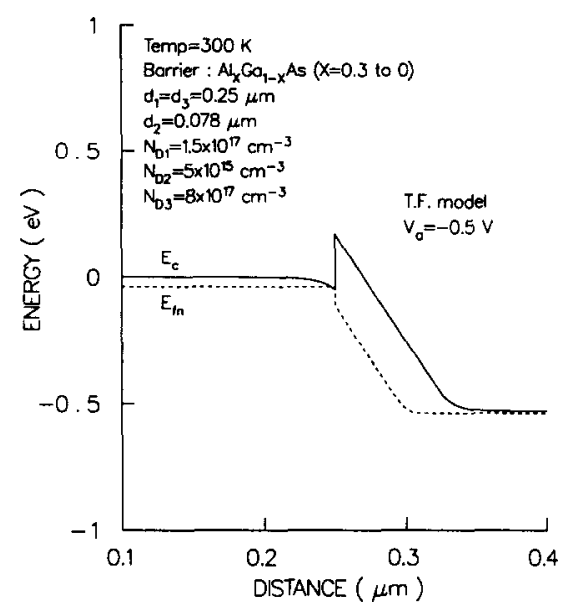

Fig. 13. Calculated conduction band profiles of the AlGaAs triangular heterojunction barrier diode using the thermionic-field emission model at a bias of (a) $0.15 \mathrm{~V}$ and (b) $-0.5 \mathrm{~V}$.

similar to those of a Schottky barrier. In Fig. 13(a) and (b), the conduction band profiles of the graded heterojunction barrier are shown at a bias of $V_{\mathrm{a}}=0.15$ and $-0.5 \mathrm{~V}$, respectively. In the forward bias, the electrons are injected from region 3 to region 1 and the barrier height is reduced as the conduction band edge of region 3 moves upward. However, in the reverse bias the electrons injecting from region 1 see an abrupt energy barrier. The electron transparent across the barrier occurs either by thermionic emission over the barrier or by tunneling through it. Since the electron energy barrier becomes more transparent under reverse bias, the tunneling process is expected to dominate as the reverse bias increascs. As shown in Fig. 12, the thermionic emission model results in much smaller current density than the experimental results in the reverse bias region. This shows that it is very difficult for electrons to gain $\sim 0.2 \mathrm{eV}$ and overcome the abrupt energy barrier from region 1. On the other hand, when the tunneling process is included, excellent agreement is obtained with the experimental results.
In Fig. 14, the measured reverse $I-V$ characteristics at different temperatures are shown with the comparison of theoretical results. The fitting to low temperature data was done by adjusting the aluminum mole fraction of heterojunction interface while maintaining the linear grading in the barrier region. Good agreement was obtained with smaller values of mole fraction of 0.27 and 0.28 for the case of $T=200$ and $180 \mathrm{~K}$, respectively (note that the aluminum mole fraction of 0.3 was used at $300 \mathrm{~K}$ ). This fact partly reflects that the conduction band edge on the GaAs side dips into the Fermi level more than estimated by the present model based on the Boltzmann distribution under high reverse bias at low temperatures. As was observed in the $n N$ heterojunctions, at low temperatures the thermionic emission model significantly underestimates the reverse current density and tunneling becomes a dominant conduction process.

The excellent agreement between theory and experiment obtained in this example indicates that the present model can be used as a powerful tool in simulating the abrupt heterojunction semiconductor devices.

\section{CONClusions}

In this paper, a numerical model is presented in which the thermionic and tunneling processes across an abrupt heterointerface are taken into account on the basis of the conventional drift-diffusion formulation. An expression of thermionic-field emission current derived from the one dimensional WKB approximation is used as a boundary condition at the heterointerface which constrains the carrier transport across the interface while maintaining the current continuity.

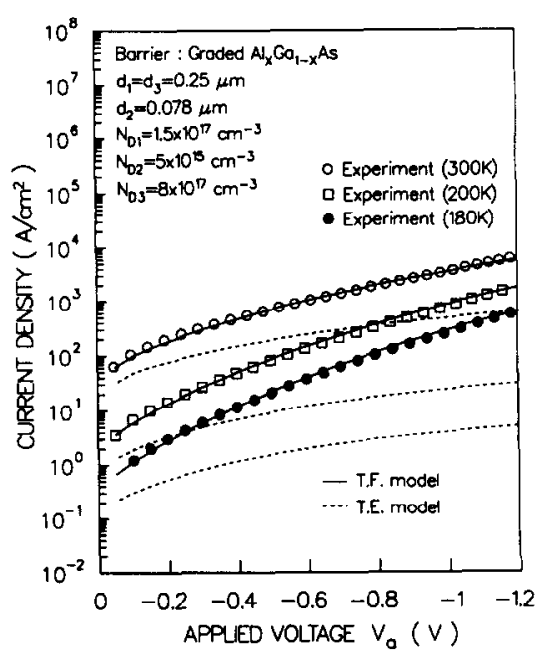

Fig. 14. The reverse $I-V$ characteristics of the AlGaAs triangular heterojunction barrier diode at different temperatures. The solid and dashed lines represent the thermionicfield emission and thermionic emission model, respectively (from the bottom, $T=180,200$ and $300 \mathrm{~K}$ ). 
By applying the present model, the $I-V$ characteristics of GaAs/AlGaAs $n N$ and $p N$ heterojunctions are analyzed as a function of device dimension, doping density, and temperature and the results are compared to those obtained by the thermionic emission model to illustrate the relative significance of both thermionic emission and tunneling mechanisms in the overall current transport. It has been confirmed that the tunneling process plays a very important role in these heterojunctions especially at high doping densities and low temperatures.

In the case of an AlGaAs triangular heterojunction barrier, the temperature-dependent $I-V$ characteristics are measured for an MBE-grown $n$-GaAs/ $\mathrm{Al}_{x} \mathrm{Ga}_{1-x} \mathrm{As} / n-\mathrm{GaAs}$ ( $x=0.25$ to 0 ) heterojunction barrier diode and good agreement is obtained between theory and experiment.

The results indicate that the model presented here will be a very useful simulation tool in modeling abrupt heterojunction devices such as HBT's and HEMT's in which the effects of thermionic emission and tunneling processes across the abrupt heterointerface should be considered in conjunction with drift-diffusion phenomena.

Acknowledgements - The authors would like to thank Dr W. $\mathrm{Li}$ and Professor P. Bhattacharya for MBE growth of the graded heterojunction barrier samples and Dr R. Kamoua for valuable discussions and measurements. This work was supported by the Army Research Office under the URI program, Contract No. DAAL03-87-K-0007.

\section{REFERENCES}

1. W. Fawcett, A. Boardman and S. Swain, J. Phys. Chem. Solids 31, 1963 (1970).
2. S. Bandyopadhyay, M. E. Klausmeier-Brown, C. M. Maziar, S. Datta and M. S. Lundstrom, IEEE Trans. Electron Devices ED-34, 392 (1987).

3. R. A. Stewart, L. Ye and J. N. Churchill, Solid-St. Electron. 32, 497 (1989).

4. M. S. Lundstrom and R. J. Schuelke, IEEE Trans. Electron Devices ED-30, 1151 (1983).

5. J. E. Sutherland and J. R. Hauser, IEEE Trans. Electron Devices ED-24, 363 (1977).

6. J. Yosida, M. Kurata, K. Morizuka and A. Hojo, IEEE Trans. Electron Devices ED-32, 1714 (1985).

7. K. Yokoyama, M. Tomizawa, H. Kanbe and T. Sudo, IEEE Trans. Electron Devices ED-30, 1283 (1983).

8. T. Ohtoshi et al., Solid St. Electron. 30, 627 (1987).

9. K. Horio and H. Yanai, IEEE Trans. Electron Devices ED-37, 1093 (1990).

10. C. B. Duke, Tunneling in Solids, p. 34. Academic Press, New York (1969).

11. C. R. Crowell and V. L. Rideout, Solid-St. Electron. 12, 89 (1969).

12. N. Chand et al., Phys. Rev. B 30, 4481 (1984).

13. D. E. Aspnes, Phys. Rev. B 14, 5331 (1976).

14. D. A. Sunderland and P. D. Dapkus, IEEE Trans. Electron Devices ED-34, 367 (1987).

15. A. Chandra and L. F. Eastman, Solid-St. Electron. 23, 599 (1980)

16. R. J. Schuelke and M. S. Lundstrom, Solid-St Electron. 27, 1111 (1984).

17. C. Y. Chang and S. M. Sze, Solid-St. Electron. 13, 727 (1970).

18. S. S. Perlman and D. L. Feucht, Solid-St. Electron. 7, 911 (1964)

19. A. A. Grinberg, M. S. Shur, R. J. Fischer and H. Morkoç, IEEE Trans. Electron Devices ED-31, 1758 (1984).

20. L. S. Lundstrom, Solid-St. Electron. 27, 49I (1984).

21. C. L. Allyn, A. C. Gossard and W. Wiegmann, Appl. Phys. Lett. 36, 373 (1980)

22. R. Kamoua, J. R. East and G. I. Haddad, J. appl. Phys. 68, 1114 (1990). 